\title{
Optical Mini-Disk Resonator Integrated into a Compact Optoelectronic Oscillator
}

\author{
P. Salzenstein*, H. Tavernier, K. Volyanskiy, N.N.T. Kim, L. Larger and E. Rubiola \\ Femto-St Institute, CNRS UMR 6174, 32 avenue de l'Observatoire, F25044 Besançon, France
}

\begin{abstract}
This work consists in the design, fabrication and characterization of mini-disk $\mathrm{MgF}_{2}$ resonators for integration into optoelectronic oscillator and first experimental results of implementation in microwave free spectral range oscillator with taper coupling optoelectronic oscillator.
\end{abstract}

PACS numbers: 06.30.Ft, 43.50.Yw, 87.64.M-, 87.57.cm

\section{Introduction}

The use of optoelectronic oscillator presents different advantages. Background comes from several years ago with some works on optoelectronic oscillator (OEO) with fiber loop [1,2]. Main advantages are low depending on delay line $\tau$. One can point out also the compatibility with telecom systems, and fact that oscillation depends on the band filter value then the carrier frequency of the oscillation can be chosen. However the system is still bulky with typically $4 \mathrm{~km}$ fiber loop, and most of all the use of the optical fiber brings difficulties with temperature regulation. That is why the choice of using an optical resonator can help to reach these problems despite the technical problems of manufacturing and coupling a mini-resonator with a fiber. We describe the reason that led to the choice of the material and the shape of the mini-resonator. Then we present first experimental results of the implementation in OEO.

\section{Choice of the optical resonator}

The optical fiber previously used in OEO is to be replaced by a whispering gallery mode (WGM) optical resonator. The coupling is realized by evanescent field. Optical signal propagates by total internal reflections inside the crystal resonator by WGM. A long delay is then reachable. One of the main advantage is that the system is compact with this resonator, thanks to a diameter in the order of few millimeters.

The expected quality factor $(Q)$ should be high. A $Q=6 \times 10^{10}$ factor was demonstrated by Grudinin and his colleagues of the Jet Propulsion Laboratory (JPL) [3]. In Table we present the advantages and disadvantages of several materials. We choose to make a mini-resonator by manufacturing a $\mathrm{MgF}_{2}$ disk, which is available as a

\footnotetext{
* corresponding author; e-mail: patrice.salzenstein@femto-st.fr
}

highly pure crystal, harder than very popular $\mathrm{CaF}_{2}$, with its 6 Mohs hardness, and easier to manipulate without damaging the surface.

TABLE

Advantages and disadvantages of several materials to be chosen for manufacturing an optical mini-resonator.

\begin{tabular}{l|c|c|c|c}
\hline \hline & $\mathrm{MgF}_{2}$ & $\mathrm{CaF}_{2}$ & Fused silica & Quartz \\
\hline $\begin{array}{l}\text { transparency } \\
\text { range }[\mu \mathrm{m}]\end{array}$ & $0.12-8.5$ & $0.2-9$ & $0.18-2.5$ & $0.19-2.9$ \\
\hline $\begin{array}{l}\text { refractive index } \\
\text { at } 1550 \mathrm{~nm}\end{array}$ & $\begin{array}{l}n_{0}=1.37 \\
n_{\mathrm{e}}=1.38\end{array}$ & $n=1.42$ & $n=1.44$ & $\begin{array}{l}n_{0}=1.54 \\
n_{\mathrm{e}}=1.53\end{array}$ \\
\hline $\begin{array}{l}\text { hardness } \\
\text { (Mohs) }\end{array}$ & 6 & 4 & $6-7$ & 7 \\
\hline crystal class & tetragonal & cubic & noncrystalline & hexagonal \\
\hline $\mathrm{H}_{2} \mathrm{O}$ pollution & good & good & bad & bad \\
\hline $\begin{array}{l}\text { mechanical } \\
\text { shock }\end{array}$ & good & bad & good & good
\end{tabular}

\section{Resonator fabrication and characterization}

First of all we have to develop a special equipment for the manufacturing of the resonator. Conception of a dedicated polishing machine is done with a $200 \mathrm{~nm}$ small eccentricity and a precision adapter. The system is hold on air-bearing support to prevent mechanical vibrations. First step is the resonator pre-shaping shown on the left of Fig. 1, with a $20^{\circ}$ bevels made by diamond grinds. Then the second step is a manual polishing shown on the right of Fig. 1 with $\mathrm{CeO}_{2}$ powder of decreasing grain size, as it is usually done with optical surfaces, and diamond powder and alumina.

Resonator is characterized by measuring Newton fringes. It shows a $1 \mathrm{~nm}$ resolution with white light phase shifting microscope. Local roughness measurements show that rugosity is of $1-5 \mathrm{~nm}$ (3-12 atoms) rms. 


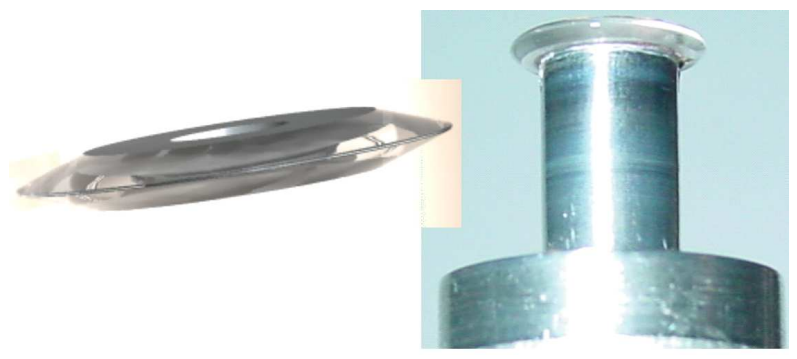

Fig. 1. Resonator pre-shaping (left) and manual polishing (right).

Unfortunately, the available instrument permits only the measurement of a small area, while a smooth defect-free surface is required in the whole equatorial region of the resonator.

\section{Taper coupling and resonance measurements}

The realized resonator is introduced in the loop thanks to a tapered fiber glued on the holder. Holder alloy and geometry match the thermal expansion of the glass. Waist is less than $3 \mu \mathrm{m}$. For coupling we use a nanopositioning system manufactured by firm LASEO in Lannion, France, with 3 -axis nanopositioning with $20 \mathrm{~nm}$ resolution.

Resonance measurements is determined with a $1550 \mathrm{~nm}$ tunable diode laser at $3 \mathrm{~mW}$ power. A $50 \mathrm{pm}$ wavelength weep that corresponds to $6 \mathrm{GHz}$ is used. A fast oscilloscope allows the analysis of very sharp phenomena as peak resonance. The oscilloscope is inserted after the photodiode that detects the optical signal coming from the mini-disk resonator coupled to the taper. Detection of the resonance peak is done in single mode excitation. Small taper size selects a thin excitation region. Wavelength span is too small to scan a full free spectral range (FSR) and scan rate is $50 \mathrm{~Hz} . Q$ factor is measured with a self homodyne method [4]. Oscillation damping gives $Q=3.4 \times 10^{8}$ and is given in Fig. 2 .

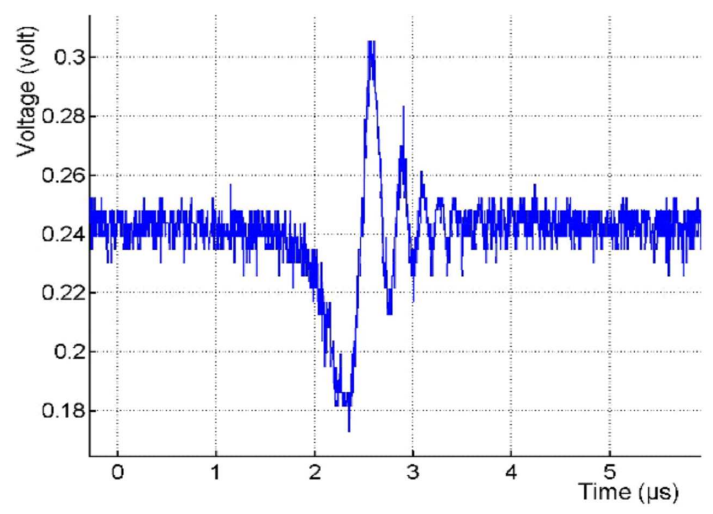

Fig. 2. $Q$ factor measurement.

\section{Implementation in $\mathrm{OEO}$ and first results}

The experimental setup consists in a classic OEO architecture. Setup and picture of the device is given in Fig. 3. The phase modulator is optically driven by a laser.

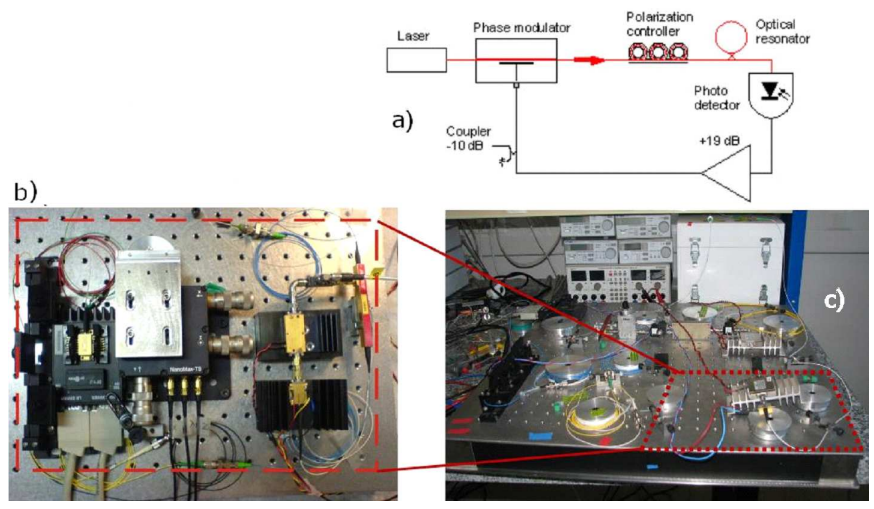

Fig. 3. Compact A3 format OEO realized with mini-resonator: (a) OEO architecture, (b) OEO with optical resonator (A3 format), (c) OEO with optical fiber loop.

The modulation microwave signal comes from the output of the microwave amplifier after crossing a $-10 \mathrm{~dB}$ directional coupler. The optical mini-resonator is coupled to the optical fiber at the output of the phase modulator. The microwave signal is amplified after the photodiode. One can notice that the size of the system is considerably decreased. Implementation of the fabricated mini-resonator led to a compact OEO that can be placed on a A3 format, to be compared to the former OEO we made with a $4 \mathrm{~km}$ fiber presented in the realized box in the bottom right figure.

The realized OEO presents enough gain and shows oscillation around $7.7 \mathrm{GHz}$. Gain of the signal coming from the coupler is measured with commercial instruments. Photonic channel transfer function is measured with a Anritsu 37369 A and oscillation signal with a Hewlett-Packard 8593E. These first results are presented in Fig. 4.

\section{Conclusion and perspectives}

In this work we made the first demonstration of the microwave-FSR oscillator with taper coupling. The presented optical channel can be used as a frequency selective component in the OEO loop. The use of the mini-resonator led to a significant reduction of the oscillator size. The vibration sensitivity of the resonator to fiber coupler prevents from oscillation.

Further work consists in measuring the phase noise on dedicated instruments developed at the laboratory $[5,6]$. It also consists in reducing or eliminating sensitivity to vibration by using a refractive index adaptator gel or other type of coupler. An improvement of the resonator $Q$ factor is expected by a better polishing of the crystal. 


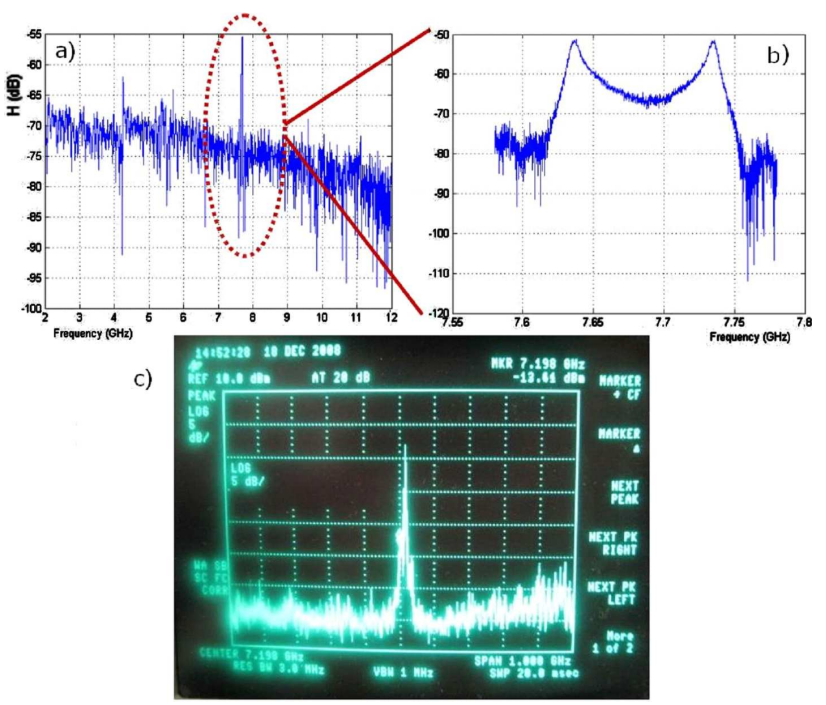

Fig. 4. First results of the photonic transfer channel function (a), (b). The gain is given in $d B$ versus frequency of the carrier in GHz. Output signal is also measured with a spectrum analyser (c).

\section{Acknowledgments}

The authors thank Dr. Gilles Cibiel from the French space agency (CNES) for his support with contract number CNES/60281/00.

\section{References}

[1] A. Neyer, E. Vosges, Appl. Phys. Lett. 40, 6 (1982).

[2] X.S. Yao, L. Maleki, J. Opt. Soc. Am. B 13, 1725 (1996).

[3] I.S. Grudinin, V.S. Ilchenko, L. Maleki, Phys. Rev. A 74, 063806(9) (2006).

[4] J. Poirson, F. Bretenaker, M. Vallet, A. Le Floch, J. Opt. Soc. Am. B 11, 2811 (1997).

[5] P. Salzenstein, J. Cussey, X. Jouvenceau, H. Tavernier, L. Larger, E. Rubiola, G. Sauvage, Acta Phys. Pol. A 112, 1107 (2007).

[6] K. Volyanskiy, J. Cussey, H. Tavernier, P. Salzenstein, G. Sauvage, L. Larger, E. Rubiola, J. Opt. Soc. Am. B 25, 2140 (2008). 